\title{
Promoting predictive, preventive and personalised medicine: European event of global importance
}

\author{
Olga Golubnitschaja • Charles Swanton • \\ Romano Danesi • Vincenzo Costigliola
}

Published online: 28 June 2011

(C) European Association for Predictive, Preventive and Personalised Medicine 2011

As The EPMA Journal has already reported, in 2010 the EU-Commission has triggered consultations with the leading experts who are actively developing innovative bio/medical technologies and promoting the field of personalised medicine. From April till June 2010, the series of workshops was signed and organised by the EUCommission as the "Vision 2020" in Brussels aiming to elaborate the items which will be fundamental to develop personalised medicine for us-European citizens in the new frame-programme Common Strategic Framework

O. Golubnitschaja $(\bowtie) \cdot$ C. Swanton $\cdot$ R. Danesi $\cdot$ V. Costigliola The "European Association for Predictive,

Preventive and Personalised Medicine",

Brussels, Belgium

e-mail: Olga.Golubnitschaja@ukb.uni-bonn.de

URL: www.epmanet.eu

O. Golubnitschaja

Department of Radiology, University of Bonn,

Bonn, Germany

C. Swanton

Translational Cancer Therapeutics Laboratory,

CR-UK London Research Institute,

London, UK

\section{R. Danesi}

Department of Internal Medicine, Division of Pharmacology, University of Pisa,

Pisa, Italy (also known as FP-8) during the next 10-15 years beginning with 2013. Upon the invitation by the EUCommission, several EPMA-experts have been involved in the creation of the items. Summarising protocols of these consultations have been published on the website of the EU-Commission.

Now it is time to approve the elaborated programme for personalised medicine in the new frame-programme. The finalising meeting "European Perspectives in Personalised Medicine" was open to all and took place in Brussels on May 12-13th 2011- see the announcement by the EUCommission in Fig. 1.

The following topics were discussed:

- The role of "omics" research and technologies in the development of personalised medicine-opportunities for clinical utility

- Biomarkers in personalised medicine: key challenges in identification, validation and qualification of safety and efficacy biomarkers

- Clinical research and trials in personalised medicineneeds and considerations

- The regulatory framework and personalised medicine: approval process towards the market and patients

- Post-approval process: cost-benefit approaches and uptake in healthcare systems

- Implementing personalised medicine: practitioner and patient perspectives, availability, awareness, resources and needs. 
Fig. 1 European event of the global meaning: "European Perspectives in Personalised Medicine" is the workshop organised by the EUCommission in order to finalise the priorities and items for personalised medicine in the new Common Strategic Framework [1]

\section{Events}

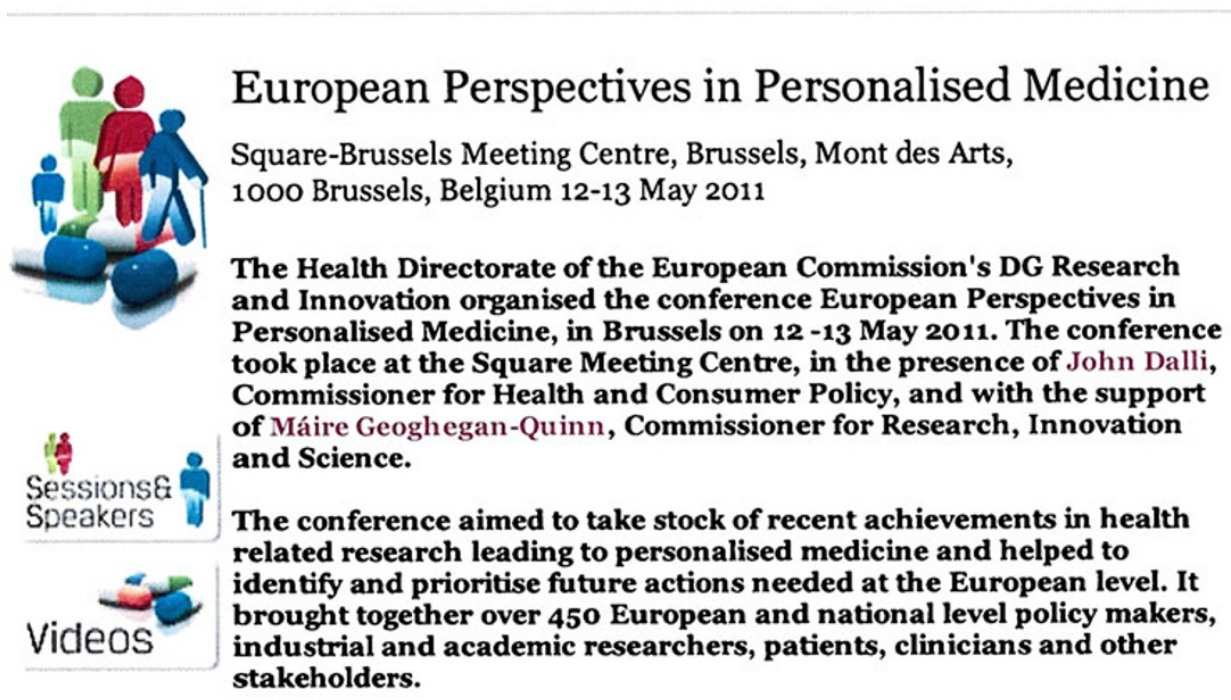

A conference summary report is available here ( $159 \mathrm{~KB})$

Please consult the list below to view conference material:

- Speakers' biographies and photos: here (1.04MB)

- Conference programme: here ( $258 \mathrm{~KB}$ )

- Conference presentations are available in the section "speakers and sessions"

- Conference videos are available in the section "videos"
In order to discuss the final items, the EUCommission has nominated representatives of the main stakeholders and leading professional groups. Among them, three of EPMA-associated experts have been invited to comment on and to contribute to the corresponding topics:

- Prof. Dr. Romano Danesi, The National EPMARepresentative in Italy,

- Prof. Dr. Charles Swanton, The National EPMABOARD in UK,

- Prof. Dr. Olga Golubnitschaja, Editor-in-Chief of The EPMA Journal \& Secretary-General of EPMA.

The audience has appreciated the contribution by the EPMA-Representatives. The key challenges, needs, topics and corresponding items promoted by EPMA for specialised calls (budgets) in the new programme, that have been taken into the final protocols by the EUCommission, are summarised below.
Innovative educational programmes for A. professionals and B. population in predictive, preventive and personalised medicine

Professionals

As Springer has very recently informed us, in Europe there are currently two international scientific journals specialised in personalised medicine, namely The EPMA Journal (EPMA / Springer) and Personalized Medicine (UK, published by "Future Medicine Ltd."). When compared to the number of journals dedicated to "omics"-technologies or medical imaging and single pathologies, this represents a very small body of literature. What are the reasons behind this?

The members of the Editorial BOARD of The EPMA Journal working weekly with the manuscripts and the peerreview procedure of the journal know very well, how difficult this task is. Different professional groups in 
personalised medicine have realised that they speak "different professional languages" less understandable for others. Consequently, great discoveries made by one professional group, are frequently underestimated or even not valued at all by the others resulting in delays to the implementation of novel developments in personalised medicine across diverse areas. Therefore, in healthcare we need to develop a new culture among experts in order to promote the multidisciplinary field of personalised medicine. Our message is-the innovative PPPM-related educational programmes for professionals should be prioritised in the Common Strategic Framework.

In order to promote innovative educational programmes, in collaboration with Springer, EPMA has developed worldwide pioneer initiatives creating the didactic materials for the field as follows:

- The EPMA Journal that regularly updates both needs and achievements in the field of PPPM in application to major and rare pathologies

- The book-series "Advances in Predictive, Preventive \& Personalised Medicine” (Book-series Editor: Olga Golubnitschaja; the book-series release is starting in 2012 with the following volumes (one of the volumes is given in the Fig. 2):

- "Healthcare Overview: New Perspectives", Volume Editor: Vincenzo Costigliola, Brussels, Belgium

- "PPPM in Diabetes mellitus", Volume Editor: Mahmood Mozaffari, Augusta, USA

- "PPPM in Neurodegenerative diseases", Volume Editor: Silvia Mandel, Haifa, Israel

- "Drug delivery systems: Advanced technologies potentially applicable in personalised medicine", Volume Editor: Jorge Coelho, Coimbra, Portugal.

This important initiative should be obligatory supported and well used at the European level and worldwide.

\section{Population}

Advanced personalisation in medicine is achievable solely in the case of participative medicine that meets the demands of patient advocacy groups focussed on individually created medical approaches. The reader will find a number of positive examples in this journal-issue concordant with this statement. Hence, the prevalence of birth asphyxia (the most frequent pathology in newborns) registered for children born to educated mothers is several times lower compared with those born to uneducated / illiterate mothers [2]. It is evident that strong restrictions in the amount of education lead to

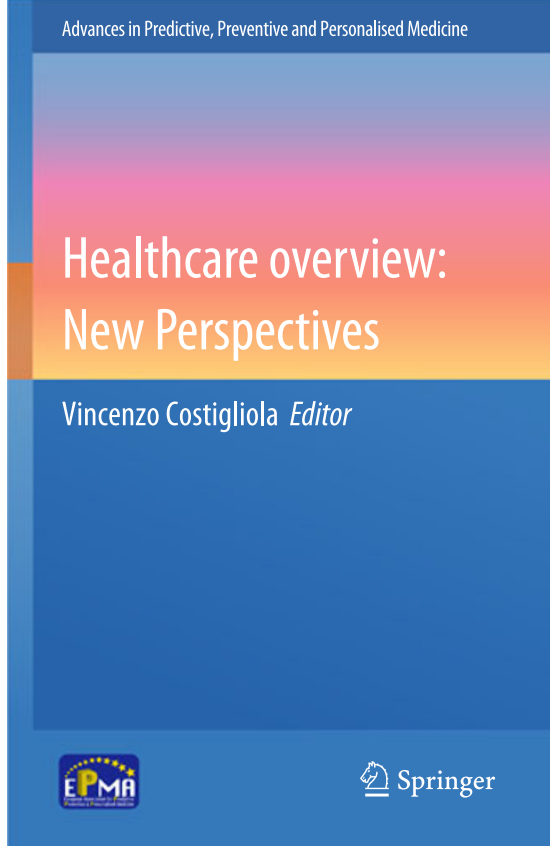

Fig. 2 Book-series "Advances in Predictive, Preventive and Personalised Medicine" in preparation by EPMA / Springer to be released in the years 2012-2015 as the didactic material for specialised educational programmes

dramatic deficits and costs that have repercussions in several branches of the society resulting in increased pressure within healthcare systems [3]. Our message isnew guidelines are essential to regulate the field in favour of educational measures for preventive programmes and advanced healthcare systems.

\section{Individualised treatment: when is the appropriate start-point?}

Current preventive and personalised strategies, consider an extremely limited spectrum of pathologies. Besides individual genetic background / predispositions of parents and history of pregnancy, the quality of birth is crucial for every newborn and decisive for plenty of potential pathologies to be developed early or later in life. These facts are currently ignored, and both the prenatal and postnatal diagnoses are performed on a very limited scale (see Figs. 3 and 4).

The obvious lack of innovative diagnostic and individualised treatment approaches applied in reproductive medicine, neonatology and paediatrics has led to a spectrum of longterm consequences in society including negative social, ethical and economic impacts. Our message is - the task of individual prediction and well-timed targeted prevention of the life-long chronic pathologies in post-natal period should 
Fig. 3 Global estimates for different countries by the World Health Organisation in 2004: newborn mortality cases per 1000 births (taken from [4])

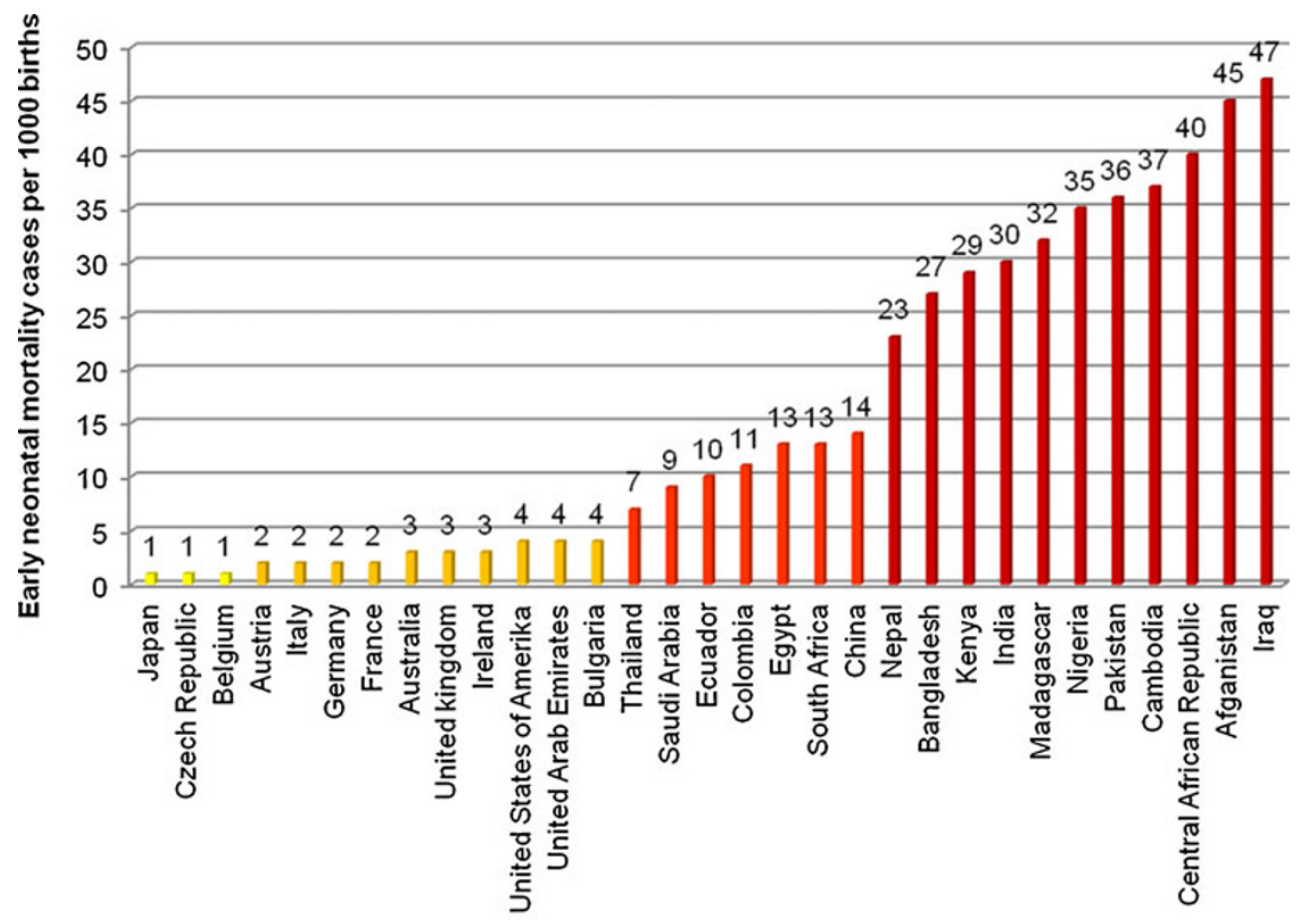

Countries

be given the extraordinary priority in advanced healthcare and related innovative programmes. New European guidelines should be elaborated emphasising advanced diagnostics approaches for reproductive medicine, healthcare of children and youth.

\section{Predicting the onset of chronic diseases and creating tailored treatments- the cost-effective medicine of the future}

As a consequence of the accumulating clinical data and knowledge about the epidemiology and pathological mechanisms of the most frequent causes of morbidity and mortality, we are currently reconsidering our view of the origins and progression of cardiovascular, oncologic and neurodegenerative diseases. The majority of these pathologies are of chronic nature: they progress from precursor lesions over one or even several decades of life until a diagnosis is made that is often too late for effective therapeutic intervention. An excellent example is the epidemic scale of diabetes mellitus type 2 witnessed in the European Union. In most industrialised countries and countries with large populations, the permanently growing cohort of diabetics creates a serious healthcare problem and dramatic health economic burden. Estimates for diabetes prevalence in the years 2030 is half a billion patients worldwide—see Fig. 5.
Fig. 4 Global estimates for newborn mortality cases per 1000 births categorised as perinatal, neonatal, stillbirth and early neonatal mortality as published by the World Health Organisation in 2004 (taken from [4])

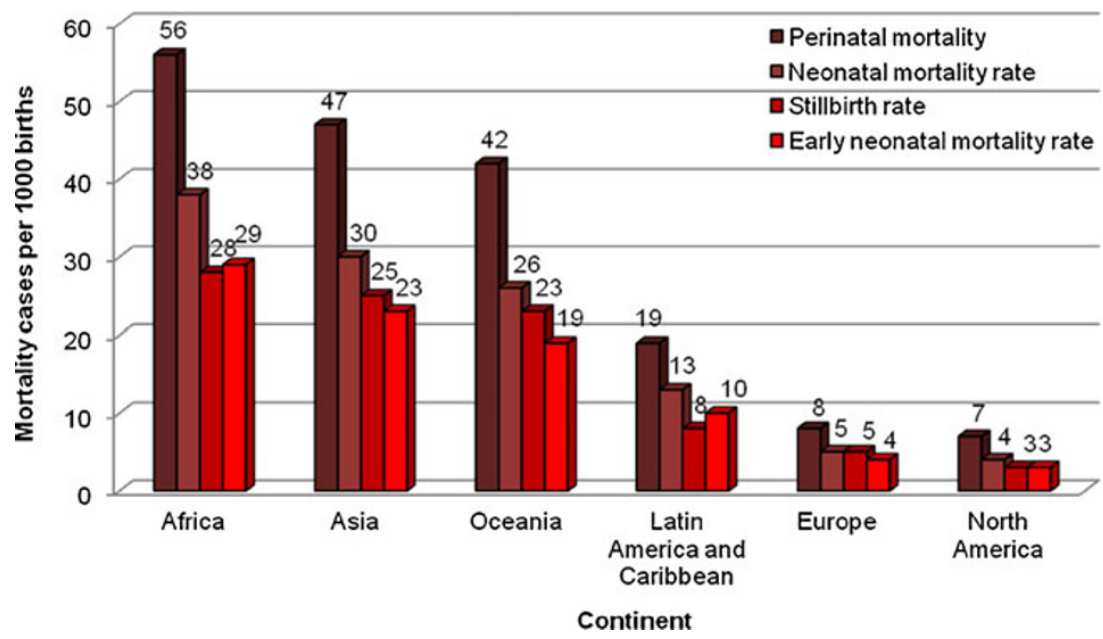


Fig. 5 Worldwide prevalence of diabetes mellitus with getting more pessimistic prognosis for the next 3 decades: current literature updates

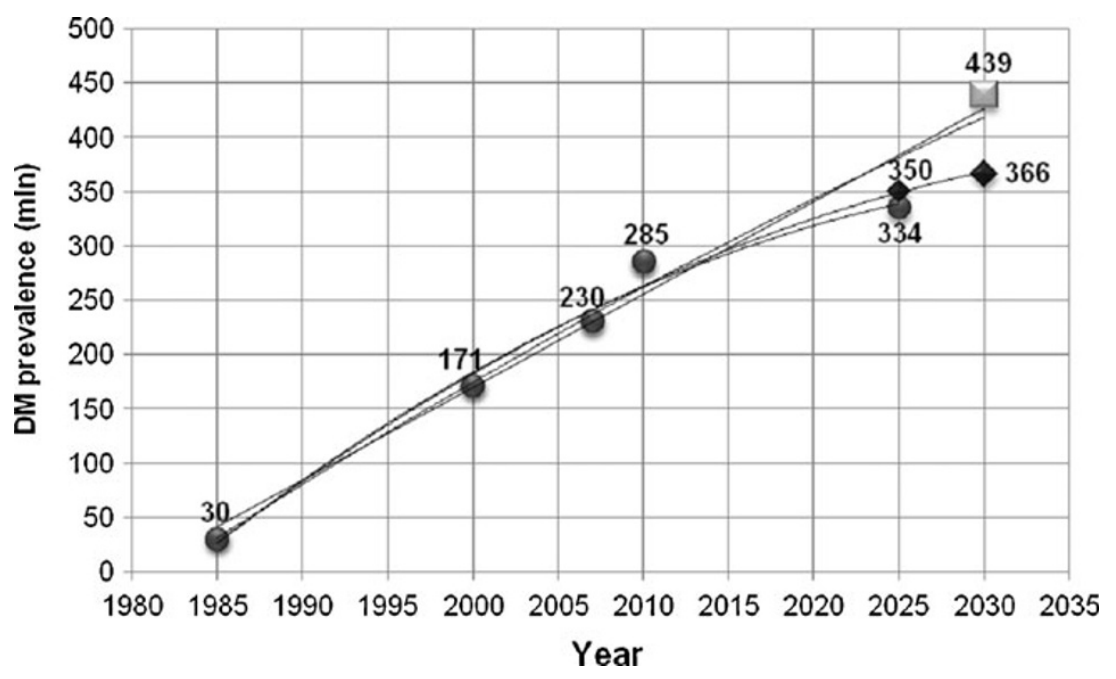

Moreover, the contemporary onset of the dominant diabetes type 2 is already observed in subpopulations of teenagers. Severe complications secondary to early onset of diabetes mellitus, such as retinopathy, nephropathy, silent ischaemia, dementia, and cancer, soon can lead to collapsing healthcare systems.

Our message is-new European guidelines should create the robust juristic and economic platform for advanced medical services utilising the cost-effective models of risk assessment followed by tailored treatments focussed on the precursor stages of chronic disease.

\section{Delivering personalised treatments in the European Union}

It is increasingly recognised that personalised medicine is essential to identify those patients most likely to benefit from novel therapeutic approaches and importantly, identify those patients likely to derive harm from a new intervention. The identification of predictive biomarkers of response to conventional and novel therapeutics is of paramount importance to minimise serious adverse events associated with treatment and maximise the proportion of patients who will derive therapeutic benefit. For the new Common Strategic Framework it is recognised that new approaches to clinical trial design and tissue biobanking procedures, combined with international academic, industry and clinical partnerships will be essential to deliver developments in personalised treatments necessary to enhance the safer and more effective delivery of novel therapeutics. As part of this, the importance of developing coordinated clinical electronic patient record and "-omics" systems for biomarker discovery, suitable for rapid implementation into clinical practice, is recognised as a key area for development within the new European initiative by the Common Strategic Framework.

\section{Future perspectives}

An optimistic versus pessimistic prognosis depends on diagnostic, preventive and treatment approaches that healthcare systems will preferably adopt in the near future. It has been predicted that in approximately 2030 the prevalence of diabetes mellitus will reach half a billion affected people worldwide, who may be additionally burdened with a spectrum of secondary complications (cancer, cardiovascular and neurodegenerative diseases). During the same period, neurodegenerative pathologies (Alzheimer's and Parkinson's diseases, glaucoma, macular degeneration, etc.) could reach more than $30 \%$ of the global disease burden. Without innovation in healthcare such developments will pose a serious threat to health economies and may lead to collapsing healthcare system.

By contrast, the effective utilisation of advanced early/ predictive diagnostics together with preventive and personalised medical approaches could enable a significant portion of the population to reach their senior years in vibrant psychosocial health, with excellent physical and mental wellbeing, participating actively in society. Global research and implementation programmes in bio/medicine, communication among scientific societies, healthcare providers, policy-makers, educators and patient organisations together with a consolidation of professional groups in the branch of personalised medicine, will play a decisive role in driving the situation in favour of one of the two scenariosan optimistic or pessimistic prognosis. The new framework aims at promotion the optimistic scenario in Europe and worldwide.

The strategic developments will be, further, discussed at the EPMA-World Congress 2011 in Bonn (Fig. 6). Everybody, who is interested in and contributing to the field is very welcome to actively participate in our meeting! 
Fig. 6 The EPMA-World

Congress in Bonn: the preliminary programme and full

congress-related information

is given at the EPMA-website:

www.epmanet.eu

\section{September $15^{\text {th }}-18^{\text {th }} 2011$}

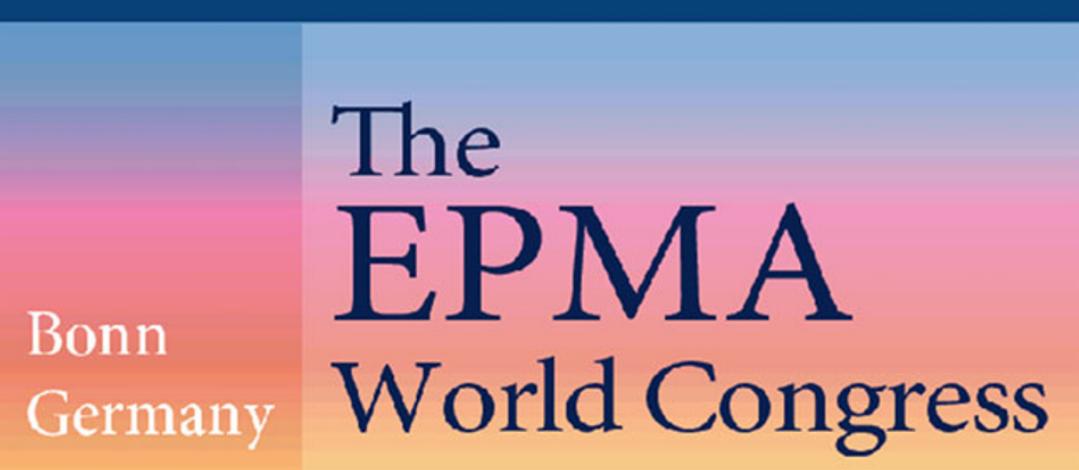

\section{References}

1. http://ec.europa.eu/research/health/events-06_en.html. Accessed 1 June 2011

2. Golubnitschaja O, Yeghiazaryan K, Cebioglu M, Morelli M, Herrera-Marschitz M. Birth asphyxia as the major complication in newborns: moving towards improved individual outcomes by

prediction, targeted prevention and tailored medical care. EPMA J. 2011. doi: 10.1007/s13167-011-0087-9

3. Golubnitschaja O, Costigliola V. Common origin but individual outcomes: time for new guidelines in personalized healthcare. Per Med. 2010;7:561-8.

4. http://whqlibdoc.who.int/publications/2007/9789241596145_eng.pdf. Accessed 1 June 2011 